\title{
Effects of subgrid-scale modeling on Lagrangian statistics in large-eddy simulation
}

\author{
Yue Yang ${ }^{\mathrm{a}}$, Guo-Wei He ${ }^{\mathrm{a}, \mathrm{b} *}$ and Lian-Ping Wang ${ }^{\mathrm{c}}$ \\ ${ }^{a}$ LNM, Institute of Mechanics, Chinese Academy of Sciences, Beijing, 100080, PR China \\ ${ }^{\mathrm{b}}$ Department of Aerospace Engineering, Iowa State University, 50011-2271 Ames, IA, USA \\ ${ }^{\mathrm{c}}$ Department of Mechanical Engineering, University of Delaware, 19716 Newark, DE, USA
}

\begin{abstract}
The application of large-eddy simulation (LES) to turbulent transport processes requires accurate prediction of the Lagrangian statistics of flow fields. However, in most existing SGS models, no explicit consideration is given to Lagrangian statistics. In this paper, we focus on the effects of SGS modeling on Lagrangian statistics in LES ranging from statistics determining single-particle dispersion to those of pair dispersion and multiparticle dispersion. Lagrangian statistics in homogeneous isotropic turbulence are extracted from direct numerical simulation (DNS) and the LES with a spectral eddy-viscosity model. For the case of longtime single-particle dispersion, it is shown that, compared to DNS, LES overpredicts the time scale of the Lagrangian velocity correlation but underpredicts the Lagrangian velocity fluctuation. These two effects tend to cancel one another leading to an accurate prediction of the longtime turbulent dispersion coefficient. Unlike the single-particle dispersion, LES tends to underestimate significantly the rate of relative dispersion of particle pairs and multiple-particles, when initial separation distances are less than the minimum resolved scale due to the lack of subgrid fluctuations. The overprediction of LES on the time scale of the Lagrangian velocity correlation is further confirmed by a theoretical analysis using a turbulence closure theory.
\end{abstract}

\section{Introduction}

Large-eddy simulation (LES) has emerged as a powerful simulation technique for fluid turbulence in general and has been applied, in particular, to study turbulent transport processes such as turbulent mixing and turbulent particle-laden flows [1,2]. The application to turbulent transport processes raises such a new requirement as that the LES with a subgrid-scale (SGS) model should correctly predict the motion of particles and the relative dispersion of particle pairs in a turbulent flow, or at least the Lagrangian statistics of the flow field [3, 4]. In LES, large-scale motions are directly computed while small-scale or SGS motions are not explicitly represented but their effects on large scales are modeled. Most of the currently existing SGS models are based on the energy budget equations: a SGS residual stress is treated as an eddy-viscosity term which dissipates the extra energy at resolved scales. Therefore, the eddy-viscosity SGS model is able to predict the energy spectra at large scales. However, it may not accurately predict other statistics which are not determined fully by the energy spectrum [5,6], such as the Lagrangian statistics. The objective of the present research is to investigate the effects of the eddy-viscosity-based SGS models on Lagrangian statistics in a turbulent flow.

*Corresponding author. Email: hgw@Inm.imech.ac.cn or guoweihe@yahoo.com

ISSN: $1468-5248$ online only

(C) 2008 Taylor \& Francis

DOI: $10.1080 / 14685240801905360$

http://www.tandf.co.uk/journals 
Lagrangian characteristics of fluid motion are of fundamental importance in turbulent transport processes: the statistics of a single-particle displacement determine the average rate of growth of a pollutant emission [7]; a particle pair dispersion characterizes the mixing rate of reactants [8]; and multiparticle dispersion statistics quantify not only the diffusion range of a patch of pollutant but also the geometry of particle clusters [9]. Therefore, it is important to study the effects of SGS modeling on Lagrangian statistics when LES is used to address turbulent transport processes. Wang et al. [10,11] use the LES with a dynamic Smagorinsky SGS model to investigate one-particle dispersion in turbulent channel flows. Armenio et al. [12] observe that, if the LES could resolve most of fluid energy, the LES with a dynamic Smagorinsky SGS model is able to accurately predict the Lagrangian statistics of a single-particle. Furthermore, they find that the twoparticle relative dispersion with initially larger separations is insensitive to the filtering operation. Our previous research [13] shows that the two-particle velocity correlations are overpredicted by the LES with an eddy-viscosity-based SGS model. In general, it is expected that the overestimation of Lagrangian velocity correlations in LES could be more pronounced when the LES resolution is poor. Wei et al. [14] proposed a hybrid Eulerian-Lagrangian LES to better simulate particle dispersion in a turbulent flow, where a conventional SGS model is used for turbulence velocity fields and a particle SGS model used for the motion of particles. There have also been other related works in this direction addressing the transport of either inertial particles or heavy particles. Armenio et al. [12] point out that the fluid particles are more sensitive to the SGS fluctuations than inertial particles. Therefore, the LES prediction can be expected to be more accurate for the inertial particles than for the fluid particles. However, the inertial particles have some additional complexity, such as settling velocity and preferential concentration. Yeh and Lei [15] and Yang and Lei [16] compared the Lagrangian statistics of LES with DNS for inertial particles and quantified the contribution of SGS motions to the settling velocity of heavy particles. Shotorban and Mashayek [17] and Kuerten [18] used the deconvolution approach to compute the Lagrangian statistics of inertial particles in turbulent channel flows. Using the deconvolution approach, the unsolved velocity fields can be partially recovered. Recently, Kuerten and Vreman [19] and Shotorban and Mashayek [20] have developed a stochastic Lagrangian particle model to better represent Lagrangian statistics of inertial particles. Fede and Simonin [21] study the contribution of SGS motions to the statistics of heavy colliding particles.

The present research focuses on the effects of the eddy-viscosity-based SGS model on Lagrangian statistics of fluid particles in isotropic turbulence. We compare the results obtained from LES with those obtained from DNS. We will first address statistics related to one-particle displacements, including the variance of one-particle displacement, one-particle Lagrangian velocity correlation and turbulence diffusivity. Next we will study the relative dispersion of particle pairs as characterized by the mean, variance and probability density functions (PDFs) of the separation distance, and two-particle Lagrangian velocity correlation. Finally, we will discuss the statistics related to multiparticle dispersion, focusing on the size and shape of four-particle clusters.

We will also attempt to develop a better understanding of the numerical results by a turbulence closure theory that predicts analytically the Taylor time micro-scales of the Lagrangian velocity correlations, starting from the governing equations in DNS and LES, respectively. The closure theory is based on the Taylor expansions of statistical functions [22] and has been used for the time correlations in DNS fields [23, 24] and Eulerian time correlations in LES fields [5] in the Navier-Stokes equations. The new development in the present paper is that we explicitly include the effects of the eddy-viscosity SGS model on the Taylor micro-scales, which confirm our numerical observation: the spectral eddy-viscosity SGS model increases the time scales of Lagrangian velocity correlations. 
The paper is organized as follows. In Section 2, the DNS and LES of isotropic turbulence will be briefly described, along with numerical methods for computing Lagrangian statistics. In Section 3, the effects of the SGS model on Lagrangian statistics of a single-particle displacement will be studied. The effects of SGS modeling on particle pair dispersion and multiparticle dispersions will be investigated in Sections 4 and 5, respectively. The analytical prediction of the Taylor microscales of Lagrangian velocity correlations will be presented in Section 6. Summary discussions and conclusions will be given in Section 7.

\section{Simulation overview}

The Navier-Stokes equations for the isotropic and homogeneous turbulence in a periodic box of side $L=2 \pi$ are written in the general form

$$
\begin{aligned}
\frac{\partial \mathbf{u}}{\partial \mathrm{t}} & =\mathbf{u} \times \omega-\nabla\left(\frac{p}{\rho}+\frac{1}{2} \mathbf{u}^{2}\right)+v \nabla^{2} \mathbf{u}+\mathbf{f}(\mathbf{x}, t), \\
\nabla \cdot \mathbf{u} & =0
\end{aligned}
$$

where $\mathbf{u}$ denotes the velocity field, $\omega \equiv \nabla \times \mathbf{u}$ the vorticity and $p$ the pressure, $\rho$ the density and $v$ the kinematic viscosity. The flow was driven by a random forcing $\mathbf{f}(\mathbf{x}, t)$ which is non-zero for the Fourier modes with the wavenumber magnitude less than 2 [25].

The DNS of the isotropic and homogeneous turbulence were performed using a standard pseudo-spectral method on a $128^{3}$ grid. The flow domain was discretized uniformly into $N^{3}$ grid points which defines the wavenumber components in Fourier space as $k_{j}=n_{j}(2 \pi / L)$, where $n_{j}=-N / 2, \ldots,-1,0,1, \ldots, N / 2-1$ for $j=1,2,3$. Aliasing errors were removed using the two-third truncation method. A stationary turbulence was generated by maintaining the constant total energy in each of the first two wavenumber shells, with the energy ratio between the two shells consistent with $k^{-5 / 3}$. The spatial resolution in spectral simulation is often monitored by the value of $k_{\max } \eta$, where $\eta \equiv(v / \epsilon)^{1 / 4}$ is the Kolmogorov length scale and the maximum wavenumber $k_{\max }$ is about $N / 3$. This quantity should be larger than unity for the Kolmogorov scale of the flow to be well resolved. The value of $k_{\max } \eta$ was typically larger than 1.4 in our simulation. The Fourier coefficients of the flow velocity were advanced in time using a second-order Adams-Bashforth method for the nonlinear term and an exact integration for the linear viscous term. The time step was chosen to ensure that the CFL number was 0.5 or less for numerical stability and accuracy.

The LES of the isotropic and homogeneous turbulence was performed on coarse grids using the same pseudo-spectral method as the DNS above. A pseudo-spectral method is chosen in present research so that the numerical dissipation is much less than the dissipation from the SGS model [26]. Therefore, the function of the SGS models is not influenced by the numerical dissipation and the effects of the SGS model on Lagrangian statistics can be correctly evaluated.

The governing equation for LES is given by

$$
\left(\frac{\partial}{\partial t}+\left[v+v_{t}\left(k \mid k_{c}\right)\right] k^{2}\right) \mathbf{u}(\mathbf{k}, t)=\mathbf{P}(\mathbf{k}) \mathcal{F}(\mathbf{u} \times \boldsymbol{\omega})+\mathbf{f}(\mathbf{k}, t),
$$

where $\mathbf{P}(\mathbf{k})=\delta_{i j}-k_{i} k_{j} / k^{2}$ and $\mathcal{F}$ denotes a Fourier transformation. A spectral eddy-viscosity SGS model [27] is used here with

$$
v_{t}\left(k \mid k_{c}\right)=v_{t}^{+}\left(k \mid k_{c}\right) \sqrt{E\left(k_{c}\right) / k_{c}},
$$


Table 1. Summary of Eulerian statistics.

\begin{tabular}{lccccc}
\hline Case & Case 1 & Case 2 & Case 3 & Case 4 & Case 5 \\
\hline Flow field type & DNS & LES & LES & DNS & LES \\
Grid number $N$ & $128^{3}$ & $64^{3}$ & $32^{3}$ & $128^{3}$ & $32^{3}$ \\
Mesh length $\mathrm{d} x$ & 0.049 & 0.098 & 0.196 & 0.049 & 0.196 \\
Viscosity $v$ & 0.010 & 0.010 & 0.010 & 0.0062 & 0.0062 \\
Taylor Reynolds number Re $\mathrm{R}_{\lambda}$ & 60 & - & - & 80 & - \\
rms fluctuating velocity $u^{\prime}$ & 0.788 & 0.774 & 0.753 & 0.822 & 0.765 \\
Dissipation rate $\epsilon$ & 0.152 & - & - & 0.169 & - \\
Spatial resolution $k_{\max } \eta$ & 2.13 & - & - & 1.45 & - \\
Kolmogorov length scale $\eta$ & 0.051 & - & - & 0.035 & - \\
Kolmogorov time scale $\tau_{\eta}$ & 0.257 & - & - & 0.192 & - \\
Eulerian integral length scale $L_{E}$ & 1.74 & 1.78 & 1.85 & 1.63 & 1.81 \\
Eddy turnover time $T_{e}$ & 2.21 & 2.30 & 2.46 & 1.98 & 2.37 \\
\hline
\end{tabular}

where

$$
v_{t}^{+}\left(k \mid k_{c}\right)=0.267+9.21 \exp \left(-3.03 k_{c} / k\right)
$$

Here, $k_{c}$ is the cutoff wavenumber in LES. When the spectral eddy-viscosity model is applied, the quantity $E\left(k_{c}\right)$ is evaluated from LES. The above SGS model is representative of the eddyviscosity-based models and it provides a satisfactory prediction of the energy spectrum. Other eddy-viscosity models share the similar properties with the above spectral eddy-viscosity SGS model. Our question is whether or not such a model could also accurately predict Lagrangian statistics in turbulent flows. The initial flow field in LES is taken directly from the corresponding DNS by applying a sharp spectral filter in order to obtain a consistent initial condition for further comparisons between DNS and LES.

Table 1 lists Eulerian statistics of the DNS and LES flow fields used in this study. $u^{\prime}=\sqrt{\frac{1}{3}\left\langle u_{i} u_{i}\right\rangle}$ is the rms turbulent fluctuation velocity. Two DNS flow Reynolds numbers are considered: $\operatorname{Re}_{\lambda}=60$ (Case 1) and $\operatorname{Re}_{\lambda}=80$ (Case 4). For the setting of Case 1, two LES runs are performed by switching on the eddy-viscosity model: the first (Case 2) uses $64^{3}$ mesh resolution and the second (Case 3) uses $32^{3}$ mesh resolution. Only one LES at $32^{3}$ mesh resolution (Case 5) is performed to match the Case 4 DNS run. Figure 1 plots the resulting energy spectra for Cases 1 , 2 and 3. It is observed from the plot that the energy spectra from the LES are in good agreement with the one from DNS at large resolved scales but decay faster than the one from DNS at small resolved scales. The latter is due to the fact that the eddy-viscosity SGS model dissipates a little bit more energy than what occurs physically at the small resolved scales. Overall, the energy spectra from the LES match well the one from the DNS, indicating that the LES in the present work is correctly implemented.

The trajectory of a fluid particle can be calculated by solving the following kinematic equation:

$$
\frac{\partial \mathbf{X}\left(\mathbf{x}_{0}, t_{0} \mid t\right)}{\partial t}=\mathbf{V}\left(\mathbf{x}_{0}, t_{0} \mid t\right)=\mathbf{u}\left[\mathbf{X}\left(\mathbf{x}_{0}, t_{0} \mid t\right), t\right]
$$

where $\mathbf{X}\left(\mathbf{x}_{0}, t_{0} \mid t\right)$ is the location at time $t$ of the fluid particle which was located at $\mathbf{x}_{0}$ at the initial time $t_{0}$, and $\mathbf{V}\left(\mathbf{x}_{0}, t_{0} \mid t\right)$ is the velocity at time $t$ of the fluid particle. Hereafter, we will use an upper-case letter to denote a Lagrangian variable and a lower-case letter for a Eulerian variable. 


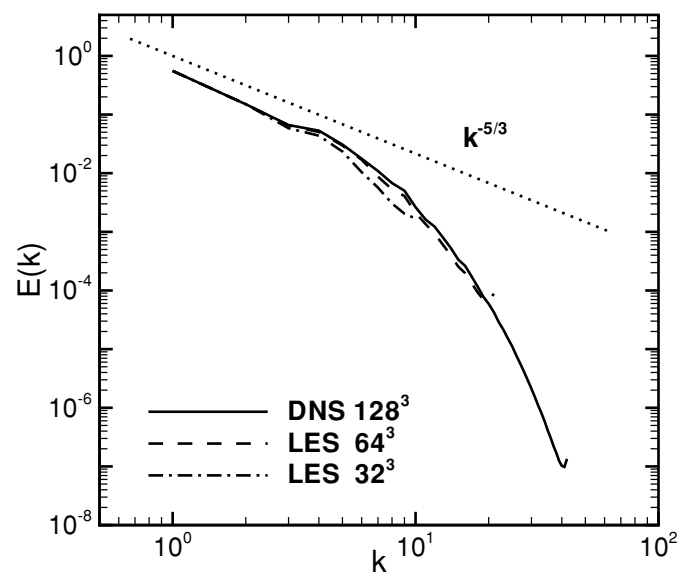

Figure 1. Energy spectra.

A three-dimensional sixth-order Lagrangian interpolation scheme is used to calculate the fluid velocity at the location of a particle [28]. The trajectory of a particle is then obtained by the explicit fourth-order Adams-Bashforth scheme. Appendix 1 provides the details of the particletracking method used in this study. To extract various Lagrangian statistics, we track the motion of an ensemble of a single particle, a particle pair and a cluster of four particles. Initially, $N_{p}$ particles are uniformly distributed in the flow domain. For each of the $N_{p}$ particles, another particle of prescribed separation is introduced for computing the statistics of particle pairs, or another three particles of prescribed separations, forming a tetrahedron together with the first one, are introduced for computing statistics of multiparticle dispersion. The Lagrangian statistics are calculated from the ensemble averaging. In addition, independent samples from different time segments can be used to further reduce the statistic fluctuations in the calculation of Lagrangian velocity correlations [29].

\section{Single-particle displacement}

In isotropic turbulence of zero mean velocity, the displacement of a single particle is positive as often as it is negative. In this circumstance, the next non-vanishing statistical moment is the variance of particle displacement, defined as

$$
\sigma_{X}^{2}(t)=\left\langle\sum_{i=1}^{3}\left(X_{i}\left(\mathbf{x}_{0}, t_{0} \mid t\right)-x_{0 i}\right)^{2}\right\rangle,
$$

which measures the displacement of a fluid particle relative to its initial position $\mathbf{x}_{0}=$ $\left(x_{01}, x_{02}, x_{03}\right)$. According to the classic theory of G. I. Taylor [7], we have two important asymptotic results

$$
\begin{cases}\sigma_{X}(t)=\sigma_{V} t, & t \ll T_{L}, \\ \sigma_{X}(t)=\sigma_{V}\left(2 T_{L} t\right)^{1 / 2}, & t \gg T_{L},\end{cases}
$$


Table 2. Summary of Lagrangian single-particle statistics.

\begin{tabular}{lccccc}
\hline Case & Case 1 & Case 2 & Case 3 & Case 4 & Case 5 \\
\hline Flow field type & $D N S$ & LES & LES & DNS & LES \\
$\mathrm{Re}_{\lambda}$ & 60 & - & - & 80 & - \\
$\sigma_{V}$ & 1.369 & 1.348 & 1.301 & 1.437 & 1.331 \\
$\sqrt{3} u^{\prime}$ & 1.365 & 1.341 & 1.304 & 1.428 & 1.325 \\
$T_{L}$ & 1.68 & 1.71 & 1.90 & 1.46 & 1.76 \\
$D_{1}$ & 3.20 & 3.18 & 3.17 & 3.24 & 3.20 \\
\hline
\end{tabular}

where $\sigma_{V}^{2}=\left\langle V_{i} V_{i}\right\rangle$ is the variance of the turbulent fluctuation velocity on a particle trajectory and $T_{L}$ is the integral time scale of one-particle, two-time velocity correlation. For a stationary, homogeneous and isotropic turbulence, $\sigma_{V}$ should be related to the Eulerian rms fluctuation velocity $u^{\prime}$ as $\sigma_{V}=\sqrt{3} u^{\prime}$. Table 2 compares the two velocity variances, showing that $\sigma_{V}$ and $\sqrt{3} u^{\prime}$ differ by less than $1.0 \%$.

Shown in Figure 2 are the standard variances of the particle displacement obtained from the DNS and LES. It is observed from the DNS that the particle displacement variance increases with time and exhibits the two asymptotic scaling behaviors. The results from LES on either fine grids or coarse grids are in good agreement with that from DNS. Therefore, the LES with the spectral eddy-viscosity SGS model could accurately predict the particle displacement variance.

The particle displacement variance is mainly determined by the variance $\sigma_{V}^{2}$ of Lagrangian fluctuation velocity and the Lagrangian correlation time $T_{L}$ [7]. The Lagrangian integral time $T_{L}$ is determined by the Lagrangian time correlation coefficient defined as

$$
\rho_{1}(\tau)=\frac{\left\langle V_{i}\left(\mathbf{x}_{0}, t_{0} \mid t_{0}\right) V_{i}\left(\mathbf{x}_{0}, t_{0} \mid t_{0}+\tau\right)\right\rangle}{\sigma_{V}^{2}},
$$

where $\tau$ is a time interval and the angle brackets denote an ensemble average over particle realizations. The repeated indices imply summation. We plot the Lagrangian time correlation

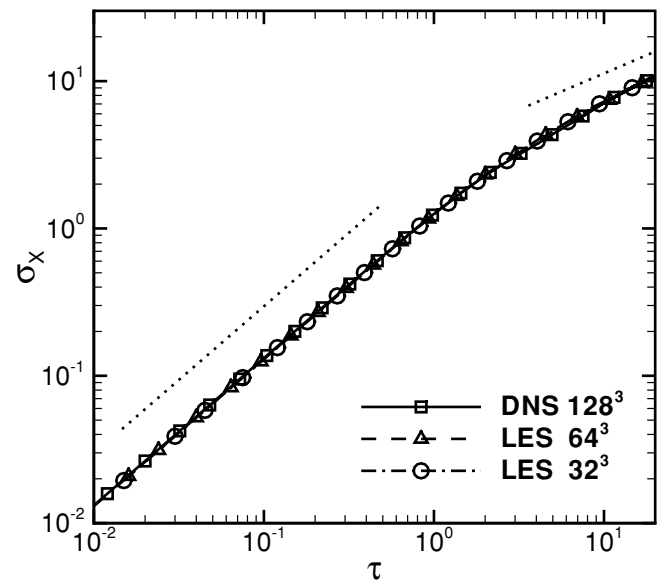

Figure 2. The standard variance of one-particle displacement. The dotted line on left bottom has an unity slope and the one on right top has a slope equal to 0.5 . 


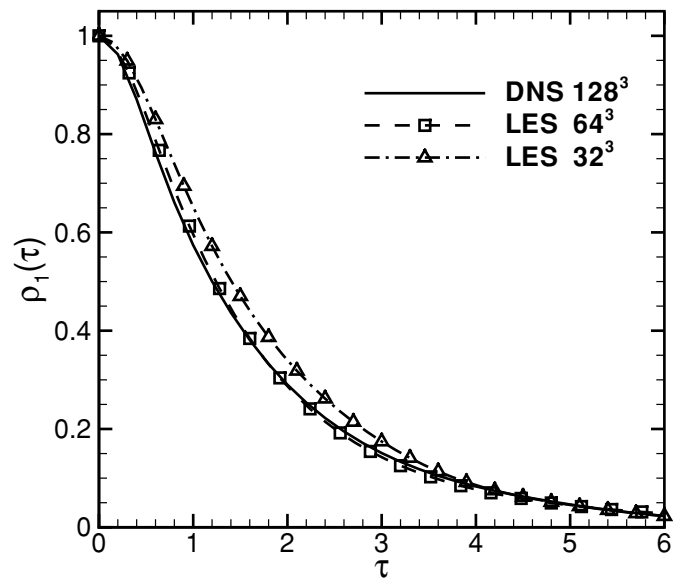

Figure 3. Lagrangian time correlation coefficients from DNS and LES at $\operatorname{Re}_{\lambda}=60$.

coefficients from DNS and LES in Figure 3. It is observed that the correlation coefficients from the LES decay more slowly than those from DNS. Moreover, the correlation coefficient from the low-resolution LES is larger than that from the high-resolution LES. The slower decays still exist when we increase the Reynolds number, see Figure 4. To quantify the observed differences, we calculate their integral scales

$$
T_{L}=\int_{0}^{\infty} \rho_{1}(\tau) \mathrm{d} \tau
$$

The ratio $T_{L}^{\mathrm{LES}} / T_{L}^{\mathrm{DNS}}$ obtained from Figure 3 is 1.02 for high-resolution LES and 1.13 for the low-resolution LES. At the higher Reynolds number $\left(\operatorname{Re}_{\lambda}=80\right)$, the integral scale ratio between the LES (Case 5) and the DNS (Case 4) is 1.21 (Table 2). These results confirm that the overprediction of the Lagrangian integral time in LES could be significant.

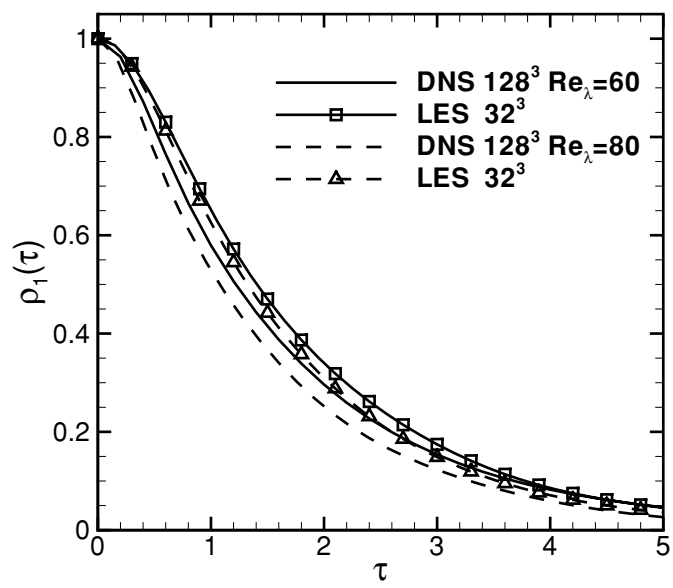

Figure 4. Lagrangian time correlation coefficients from DNS and LES at $\operatorname{Re}_{\lambda}=60$ and $\operatorname{Re}_{\lambda}=80$. 
In LES, the SGS motions are not explicitly represented and large-scale motions are modified by the eddy-viscosity SGS model. As a result, the velocity fields in LES are more coherent and thus more correlated than those in DNS. Therefore, the Lagrangian velocity correlation in LES decays more slowly than that in DNS. We further calculate the longtime diffusion coefficients from the DNS and LES

$$
D_{1}=\frac{1}{2} \frac{\mathrm{d}}{\mathrm{dt}}\left(\sigma_{X}^{2}\right), \quad t \gg T_{L}
$$

Interestingly, Table 2 shows that the longtime dispersion coefficients in LES are only slightly less than those in DNS. This can be explained as follows: while the integral time scale $T_{L}$ is overpredicted by up to $13.1 \%$ in LES, the velocity fluctuation $\sigma_{V}$ is actually underpredicted by up to $5.0 \%$ in LES. These two errors tend to cancel each other, leading to a rather accurate prediction of the longtime single-particle turbulent diffusivity $D_{1}=\sigma_{V}^{2} T_{L}$.

\section{Particle pair dispersion}

A particle pair in a turbulent flow on average moves apart from each other, as illustrated in Figure 5. As a result, its mean separation distance always tends to increase. The relative dispersion is dependent on the turbulent velocity fields and its initial separation distances. In DNS, the velocity fields are fully resolved at all scales and the contributions to relative dispersion from fluid motions at all scales are well represented. However, in LES, the large-scale velocity field is modified by the SGS models and the small-scale fluid motion is completely missing, which may significantly affect the relative dispersion of particle pairs at unresolved scales. The influences can be measured by the statistics of relative separations and velocities.
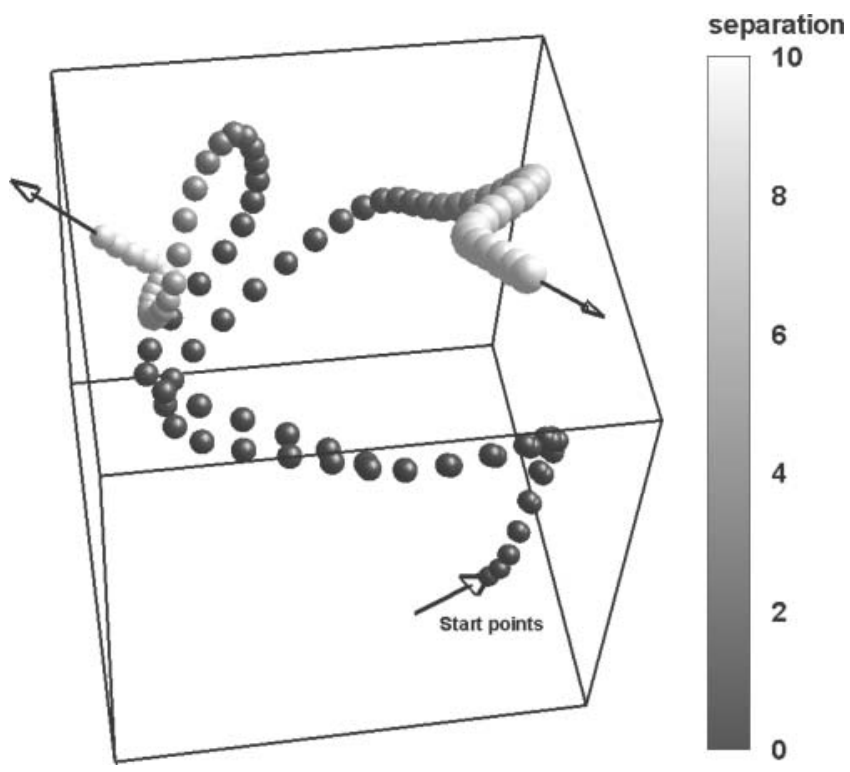

Figure 5. A pair particle separation with an initial separation distance equal to the Kolmogorov scale. 
The relative separation vector $\mathbf{R}=\left(R_{1}, R_{2}, R_{3}\right)$ between a particle pair at one time is denoted by

$$
\mathbf{R}\left(\mathbf{r}, t_{0} \mid \tau\right)=\mathbf{X}\left(\mathbf{x}_{0}, t_{0} \mid t_{0}+\tau\right)-\mathbf{X}\left(\mathbf{x}_{0}+\mathbf{r}, t_{0} \mid t_{0}+\tau\right)
$$

where $\mathbf{r}$ is the initial separation vector between a particle pair and $\tau$ is the time delay. Since the statistics of $\mathbf{R}$ is independent of initial location $\mathbf{x}_{0}$, we drop off $\mathbf{x}_{0}$ in $\mathbf{R}$ to simplify the notation. Thus, the separation distance is calculated from

$$
R\left(\mathbf{r}, t_{0} \mid \tau\right)=\sqrt{\mathbf{R}\left(\mathbf{r}, t_{0} \mid \tau\right) \cdot \mathbf{R}\left(\mathbf{r}, t_{0} \mid \tau\right)}
$$

and their mean and variance are calculated from

$$
\begin{aligned}
& m_{2}(r, \tau)=\left\langle R\left(\mathbf{r}, t_{0} \mid \tau\right)\right\rangle \\
& \sigma_{2}^{2}(r, \tau)=\left\langle\left[R\left(\mathbf{r}, t_{0} \mid \tau\right)-\left\langle R\left(\mathbf{r}, t_{0} \mid \tau\right)\right\rangle\right]^{2}\right\rangle=\left\langle R_{i}\left(\mathbf{r}, t_{0} \mid \tau\right) R_{i}\left(\mathbf{r}, t_{0} \mid \tau\right)\right\rangle-m_{2}^{2}(r, \tau)
\end{aligned}
$$

where $r$ is the magnitude of the initial separation vector $\mathbf{r}$. The mean and variance are dependent only on the separation distance $r$ and time delay $\tau$ since homogeneity and isotropy are assumed.

From Equation (13), we can obtain the mean-squared separation distance as follows:

$$
\left\langle R_{i}\left(\mathbf{r}, t_{0} \mid \tau\right) R_{i}\left(\mathbf{r}, t_{0} \mid \tau\right)\right\rangle=m_{2}^{2}(r, \tau)+\sigma_{2}^{2}(r, \tau) .
$$

On the other hand, we can formulate the mean-squared separation distance in terms of the Lagrangian velocity

$$
\begin{aligned}
\left\langle R_{i}\left(\mathbf{r}, t_{0} \mid \tau\right) R_{i}\left(\mathbf{r}, t_{0} \mid \tau\right)\right\rangle= & r^{2}+2 \int_{t_{0}}^{t_{0}+\tau} \int_{t_{0}}^{t_{0}+\tau}\left[\left\langle V_{i}\left(\mathbf{x}_{0}, t_{0} \mid s^{\prime}\right) V_{i}\left(\mathbf{x}_{0}, t_{0} \mid s\right)\right\rangle\right. \\
& \left.-\left\langle V_{i}\left(\mathbf{x}_{0}, t_{0} \mid s^{\prime}\right) V_{i}\left(\mathbf{x}_{0}+\mathbf{r}, t_{0} \mid s\right)\right\rangle\right] \mathrm{d} s^{\prime} \mathrm{d} s
\end{aligned}
$$

The second term of the integration in Equation (15) can be represented as

$$
\begin{aligned}
& \left\langle V_{i}\left(\mathbf{x}_{0}, t_{0} \mid s^{\prime}\right) V_{i}\left(\mathbf{x}_{0}+\mathbf{r}, t_{0} \mid s\right)\right\rangle \\
& =\int\left\langle\delta\left(\mathbf{r}^{\prime}-\mathbf{R}\left(\mathbf{r}, t_{0} \mid s^{\prime}\right)\right) V_{i}\left(\mathbf{X}\left(\mathbf{x}_{0}, t_{0} \mid s^{\prime}\right), s^{\prime} \mid s^{\prime}\right) V_{i}\left(\mathbf{X}\left(\mathbf{x}_{0}, t_{0} \mid s^{\prime}\right)+\mathbf{r}^{\prime}, s^{\prime} \mid s\right)\right\rangle \mathrm{d} \mathbf{r}^{\prime} \\
& =\int\left\langle\delta\left(\mathbf{r}^{\prime}-\mathbf{R}\left(\mathbf{r}, t_{0} \mid s^{\prime}\right)\right)\right\rangle\left\langle V_{i}\left(\mathbf{X}\left(\mathbf{x}_{0}, t_{0} \mid s^{\prime}\right), s^{\prime} \mid s^{\prime}\right) V_{i}\left(\mathbf{X}\left(\mathbf{x}_{0}, t_{0} \mid s^{\prime}\right)+\mathbf{r}^{\prime}, s^{\prime} \mid s\right)\right\rangle \mathrm{d} \mathbf{r}^{\prime} \\
& =\int P\left(r \mid r^{\prime}, s^{\prime}\right)\left\langle V_{i}\left(\mathbf{x}_{0}, s^{\prime} \mid s^{\prime}\right) V_{i}\left(\mathbf{x}_{0}+\mathbf{r}^{\prime}, s^{\prime} \mid s\right)\right\rangle \mathrm{d} \mathbf{r}^{\prime},
\end{aligned}
$$

where $P\left(r \mid r^{\prime}, s^{\prime}\right)$ denotes the probability density function (PDF) of the separation distance $r^{\prime}$ at time $s^{\prime}$ of the particle pair whose separation is $r$ at the initial time $t_{0}$. The first and third steps in this development introduce the different fluid particles as the labeling points due to homogeneity. The second step invokes Corrsin's independent hypothesis [30], stating that the particle separation PDFs at a large time are independent of the particle-pair spacetime velocity correlations, and in isotropic turbulence, the PDF of separation vector can be equivalently represented by the PDF 
of separation distance. The independence hypothesis has been investigated theoretically [31] and numerically [32]. Since the PDF is independent of initial time, we will drop off the initial time $t_{0}$ from the PDF and denote it as $P\left(r \mid r^{\prime}, s^{\prime}\right)$.

Finally, we obtain the mean-squared separation distance as follows:

$$
\begin{aligned}
& \left\langle R_{i}\left(\mathbf{r}, t_{0} \mid \tau\right) R_{i}\left(\mathbf{r}, t_{0} \mid \tau\right)\right\rangle \\
& \quad=r^{2}+2 \sigma_{V}^{2} \int_{t_{0}}^{t_{0}+\tau} \int_{t_{0}}^{t_{0}+\tau}\left[\rho_{1}\left(s-s^{\prime}\right)-\int P\left(r \mid r^{\prime}, s^{\prime}\right) \rho_{2}\left(r^{\prime}, s-s^{\prime}\right) \mathrm{d} r^{\prime}\right] \mathrm{d} s^{\prime} \mathrm{d} s,
\end{aligned}
$$

where $\sigma_{V}^{2}=\left\langle V_{i} V_{i}\right\rangle$ is again the variance of Lagrangian fluctuation velocities, and the Lagrangian space time velocity correlation at two times of a particle pair is defined as

$$
\rho_{2}(r, \tau)=\left\langle V_{i}\left(\mathbf{x}_{0}, t_{0} \mid t_{0}\right) V_{i}\left(\mathbf{x}_{0}+\mathbf{r}, t_{0} \mid t_{0}+\tau\right)\right\rangle / \sigma_{V}^{2}
$$

Therefore, Equation (17) indicates that the mean-squared separation distance depends on the one-particle Lagrangian velocity correlation $\rho_{1}\left(s-s^{\prime}\right)$, two-particle Lagrangian velocity correlation $\rho_{2}\left(r^{\prime}, s-s^{\prime}\right)$, and the PDF, $P\left(r \mid r^{\prime}, s^{\prime}\right)$, of separation distance. We will calculate these quantities from DNS and LES for comparison in this section. Another useful quantity is the one-time Lagrangian velocity correlation of particle pairs [32], defined by

$$
\rho_{r}(r, \tau)=\left\langle V_{i}\left(\mathbf{x}_{0}, t_{0} \mid t_{0}+\tau\right) V_{i}\left(\mathbf{x}_{0}+\mathbf{r}, t_{0} \mid t_{0}+\tau\right)\right\rangle / \sigma_{V}^{2} .
$$

This quantity describes the statistic property of the relative velocity of a particle pair. We will also calculate it to investigate the Lagrangian statistics in LES.

To calculate Lagrangian spacetime correlations, particles are initially released in pairs with different separation distances. A half number of particles were first placed in a flow domain, and then the other half number of particles were placed a certain distance away from the previous ones. In the present calculation, the total particle number $N_{p}$ is fixed and those particles are divided into $n_{p}$ groups in terms of their initial separations $0, r_{0}, 2 r_{0}, \ldots,\left(n_{p}-1\right) r_{0}$, so each group has $N_{p} / n_{p}$ particles. Here, the maximum initial separation is compared to the half of the periodic side length $L$ and $r_{0} \approx L / 2 n_{p}$ is the interval between initial separations. As a result, if $n_{p}$ is large, there are not enough samples in each group for a good statistical averaging which may cause severe wiggling of the contours. On the other hand, if $n_{p}$ is too small, there may not be a sufficient number of data points to construct the correlation surface leading to visual discontinuity of the contours. Therefore, due to the current storage capacity of computer, we need to carefully choose the total number of particles $N_{p}$ and the group number $n_{p}$. The total particle number $N_{p}$ in the calculation of the spacetime correlations was 64 times of that in the calculation of one-particle statistics and the group number is $n_{p}=64$.

Figure 6 shows the temporal evolutions of the separation variances of the particle pair for five different initial separations $r / \eta=0.25,1,4,16,32$. The DNS results confirm the observations of Yeung [32]: the separation variances initially grow as time increases, with the slope equal to 2 on a log-log plot. At long times, the variances grow linearly with time. In between, there exist finite transient ranges which are shorter for larger initial separations than smaller initial separations. The LES qualitatively reproduces the same results. However, the separation variances in LES grow more slowly than those in the DNS. Namely, the LES somewhat underpredicts the separation variances. The underprediction is more significant for smaller initial separations than larger initial separations. We also plot the mean separation distance in Figure 7, which 


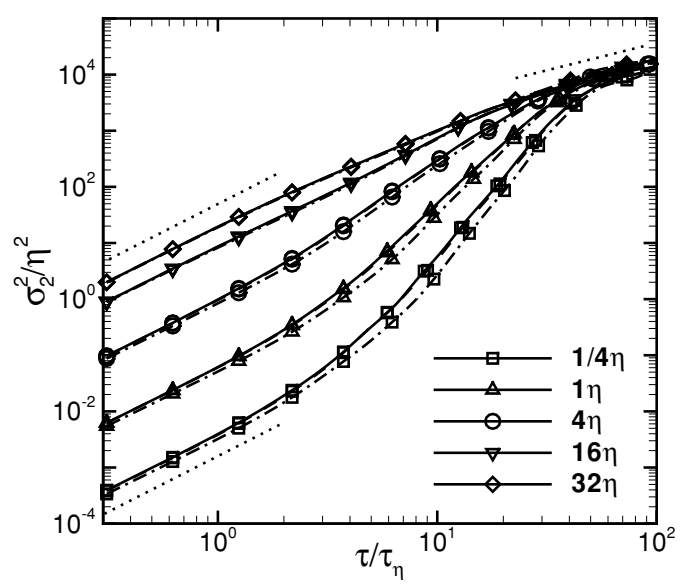

Figure 6. Variances of separation distances of particle pairs for different initial separation distances: solid lines DNS $128^{3}$, dashed lines LES $64^{3}$ and dash-dotted lines LES $32^{3}$. The symbols are corresponding to the different initial separations where $\eta$ denotes the Kolmogorov length scale. The left two dotted straight lines have the slopes equal to 2 and the right top one has a unity slope.

shows that it takes a longer time for the LES field to separate a particle pair than the DNS field, again showing that the LES field disperses the particle pair less efficiently than the DNS field.

Figure 8 plots the contours of the Lagrangian spacetime correlations from the DNS at $\operatorname{Re}_{\lambda}=60$ and the corresponding LES. It shows that the contours from the LES on coarse grid $\left(32^{3}\right)$ are clearly larger than the DNS but those from the LES on fine grid $\left(64^{3}\right)$ are only slightly larger than the DNS. Therefore, the Lagrangian spacetime correlation coefficient in LES decays more

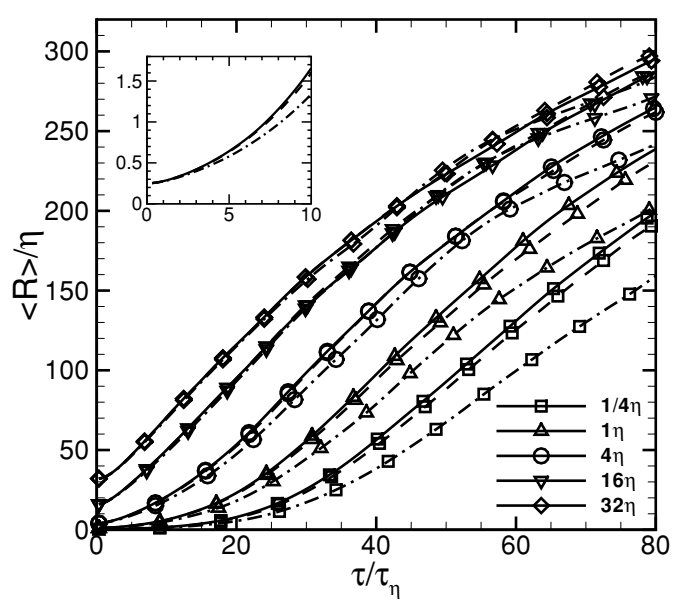

Figure 7. Means of separation distances of particle pairs for different initial separation distances. In the inset, the evolution of average separation distance with $r=0.25 \eta$ at small times: solid lines DNS $128^{3}$, dashed lines LES $64^{3}$ and dash-dotted lines LES $32^{3}$. Different symbols are corresponding to the different initial separations where $\eta$ denotes the Kolmogorov length scale. 


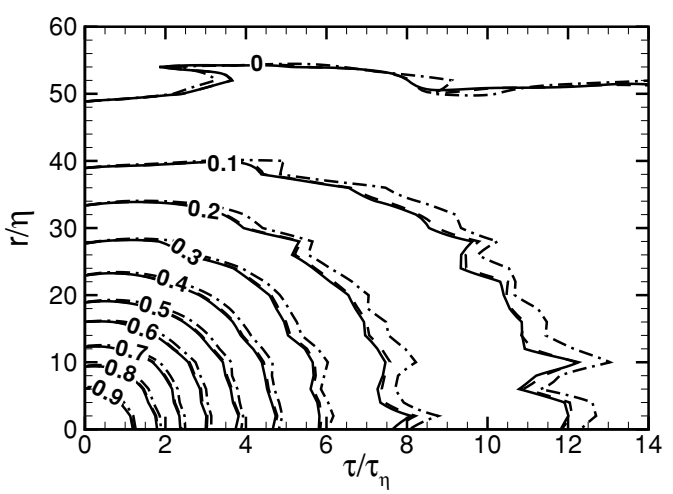

Figure 8. Contours of Lagrangian spacetime correlation coefficients: solid lines DNS $128^{3}$, dashed lines LES $64^{3}$ and dash-dotted lines LES $32^{3}$.

slowly than that of DNS. Some wiggles are observed on the iso-correlation contours in Figure 8 and the PDF lines in Figure 10. They are due to insufficient samples but do not affect the conclusions obtained. It is not feasible to improve the convergence within our current computer capability.

To further support the above observations, we plot the temporal evolutions of the one-time Lagrangian velocity correlation coefficients of particle pairs for five different initial separations in Figure 9. The results from the DNS are in agreement with the study of Yeung [32]: the correlation coefficients are steadily deceasing as time $\tau$ increases, with the exception that the correlation coefficients for the initially larger separations increase slightly at short time before decreasing. The correlation coefficients for small initial separations do not exhibit such short-time behavior, but decrease monotonically with time starting from a nearly perfect correlation. The LES data qualitatively reproduce the same trend as the DNS one. However, the

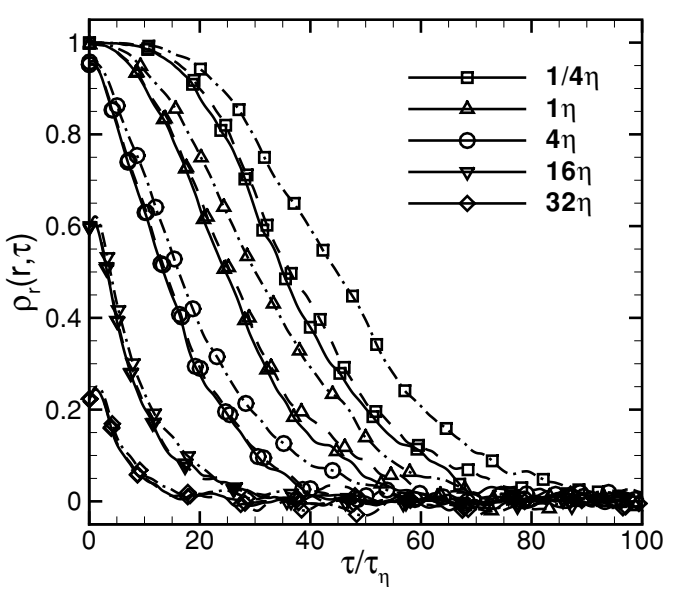

Figure 9. The one-time Lagrangian velocity correlation coefficients of particle pairs for different initial separations: solid lines DNS $128^{3}$, dashed lines LES $64^{3}$ and dash-dotted lines LES $32^{3}$. The symbols are corresponding to the different initial separations and $\eta$ denotes the Kolmogorov length scale. 
correlation coefficients from LES decay more slowly than those from DNS. The slower decay is evidently more noticeable for the initially smaller separations than for the initially larger separations. A possible explanation for the slower decay is that the Lagrangian velocity field in LES is more correlated than that in DNS. In LES, the exclusion of small-scale motions makes the LES velocity fields less intermittent. Meanwhile, the spectral eddy-viscosity SGS model plays a role of dissipation which reduces the fluctuations of LES velocity fields more intensively than the molecular dissipation alone. Both the exclusion of small-scale motions and the eddy-viscosity SGS model lead to an overprediction of Lagrangian velocity correlation coefficients.

The PDF of the separation distance is another factor affecting the mean-squared separation. The PDF evolves from an initial delta distribution to a final Chi-square distribution [32]. Figure 10 displays the PDF of the separation distance for four different initial separations at a given time. Since the PDFs evolve progressively, the temporal evolution of the PDF with a given initial separation could be typically represented by the PDF behavior at one later time with different initial separations. Therefore, Figure 10 can roughly correspond to the evolution of the PDF, for a particle pair with a small initial separation, at several later times. It is observed

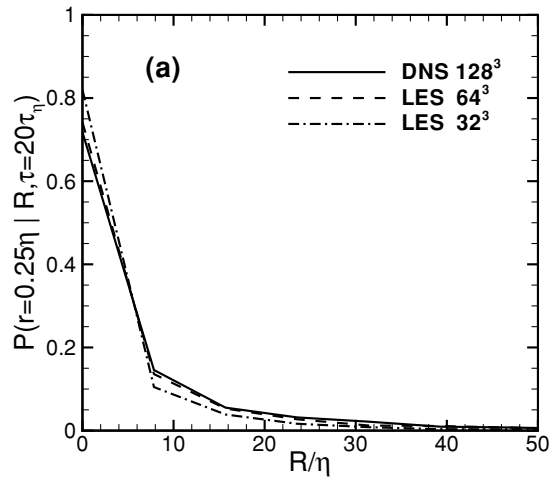

(a) $r=\frac{1}{4} \eta$.

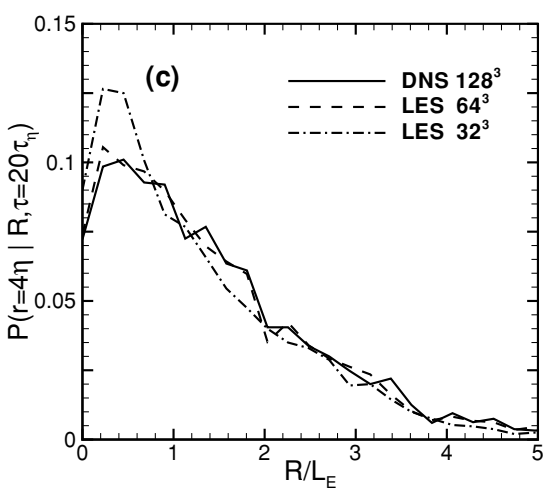

(c) $r=4 \eta$.

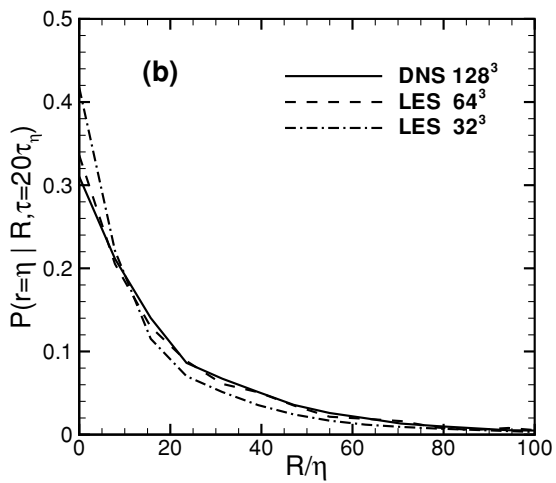

(b) $r=\eta$.

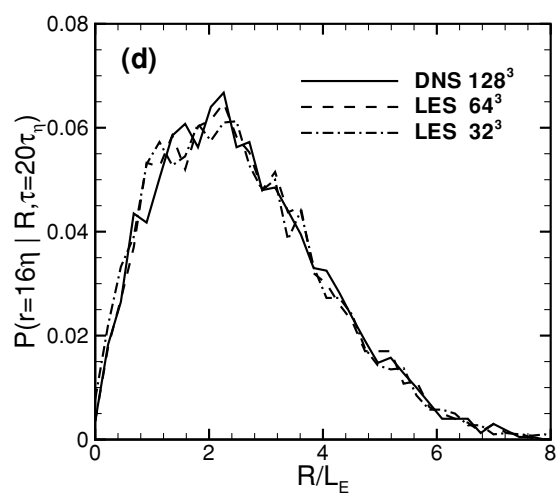

(d) $r=16 \eta$.

Figure 10. The PDFs of the separation distances for the different initial separations $r$ at $\tau=20 \tau_{\eta}$. (a) $r=\frac{1}{4} \eta$, (b) $n=\eta$, (c) $r=4 \eta$, (d) $r=16 \eta$. 
that the LES predicts the shapes of the PDFs from the initial delta distribution to the chisquare one. However, the LES exhibits a higher peak and a lower tail than the DNS from early to later stages, although it finally approaches the DNS results. This can be understood as follows: the absence of small-scale motions in LES leads to slower increase in separation for particle pairs at small separations than in DNS where the small-scale velocity could be dominating at small separations. As a result, it takes a longer time for a particle pair to move away from each other in LES. As soon as the particle separation grows beyond the cutoff scale, the rate of increase in pair separation in LES is expected to be essentially the same as that in DNS, since the large-scale velocity dominates the relative dispersion at this stage of larger separations.

Those observations are consistent with the implication of Equation (17). As observed in Figure 3, the quantity $\rho_{1}$ in LES is slightly larger than that in DNS; in Figures 8 and 10, in comparison with the DNS, $\rho_{2}$ in LES is larger and has a larger weight on small $r$, where the PDF $P$ plays a role of weight. As a result, the mean-squared separation distance in LES should be smaller than that in DNS, according to Equation (17). The conclusion can be again confirmed via Figures 6 and 7 in terms of Equation (14).

\section{Multiparticle dispersion}

Multiparticle dispersion describes the evolution of particle clusters of a particular shape and provides more information on turbulent transport processes than either one-particle or twoparticle dispersion. As discussed before, while single-particle displacement characterizes the average rate of growth of a pollutant emission from a fixed source location and a particle pair dispersion characterizes the mixing rate of pollutants a multiparticle dispersion characterizes the size and shape evolutions of particle clusters. Here we will study the time evolution of a tetrahedron of four particles. Due to the vortex stretching and small-scale intermittency in turbulent flows, an initially regular tetrahedron of four equilateral triangles could be progressively distorted into an asymptotic coplanar object, such as a pancake or needle-like one. The distortion leads to size increasement and shape change of the tetrahedrons, see Figure 11. We shall investigate the LES prediction on the size and shape evolutions of a tetrahedron in turbulent flows.

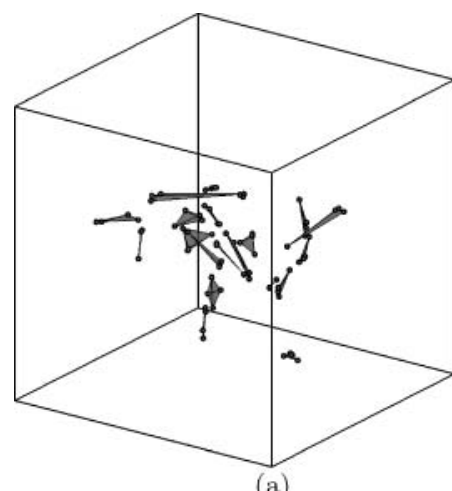

(a)

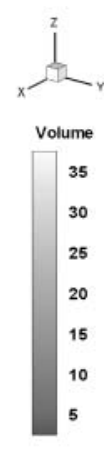

Figure 11. The snapshots of initial regular tetrahedrons of side length equal to the Kolmogorov length scale. (a) The snapshot at $\tau=40 \tau_{\eta}$. (b) The snapshot at $\tau=70 \tau_{\eta}$. 
The size of a tetrahedron can be measured by its surface area and volume. Given one particle at $\mathbf{X}_{0}^{(1)}=\left(x_{0}, y_{0}, z_{0}\right)$ in a flow field at the initial time, the other three particles are placed at

$$
\begin{aligned}
& \mathbf{X}_{0}^{(2)}=\left\{x_{0}-\frac{\sqrt{3}}{6} \eta, y_{0}-\frac{1}{2} \eta, z_{0}-\frac{\sqrt{6}}{3} \eta\right\}, \\
& \mathbf{X}_{0}^{(3)}=\left\{x_{0}-\frac{\sqrt{3}}{6} \eta, y_{0}+\frac{1}{2} \eta, z_{0}-\frac{\sqrt{6}}{3} \eta\right\}, \\
& \mathbf{X}_{0}^{(4)}=\left\{x_{0}+\frac{\sqrt{3}}{3} \eta, y_{0}, z_{0}-\frac{\sqrt{6}}{3} \eta\right\} .
\end{aligned}
$$

These four particles form a regular tetrahedron of side length equal to the Kolmogorov length scale $\eta$. The area of the triangle face formed by the first three particles can be calculated as follows:

$$
\begin{aligned}
S^{(123)}= & \frac{1}{2} \sqrt{ }\left[\left(y^{(2)}-y^{(1)}\right)\left(z^{(3)}-z^{(1)}\right)-\left(z^{(2)}-z^{(1)}\right)\left(y^{(3)}-y^{(1)}\right)\right]^{2} . \\
& +\left[\left(z^{(2)}-z^{(1)}\right)\left(x^{(3)}-x^{(1)}\right)-\left(x^{(2)}-x^{(1)}\right)\left(z^{(3)}-z^{(1)}\right)\right]^{2} \\
& +\left[\left(x^{(2)}-x^{(1)}\right)\left(y^{(3)}-y^{(1)}\right)-\left(y^{(2)}-y^{(1)}\right)\left(x^{(3)}-x^{(1)}\right)\right]^{2},
\end{aligned}
$$

where the superscripts ' 123 ' denote the particles forming the triangle face. The areas of other faces, such as $S^{(124)}, S^{(134)}$ and $S^{(234)}$ can be calculated in the same way as $S^{(123)}$. Therefore, the surface area of the tetrahedron is

$$
S=S^{(123)}+S^{(124)}+S^{(134)}+S^{(234)} .
$$

In terms of particle locations, we can easily calculate the volume of the tetrahedron formed by $\mathbf{X}^{(1)}, \mathbf{X}^{(2)}, \mathbf{X}^{(3)}$ and $\mathbf{X}^{(4)}$

$$
V=\frac{1}{6}\left|\begin{array}{lll}
x^{(2)}-x^{(1)} & x^{(3)}-x^{(1)} & x^{(4)}-x^{(1)} \\
y^{(2)}-y^{(1)} & y^{(3)}-y^{(1)} & y^{(4)}-y^{(1)} \\
z^{(2)}-z^{(1)} & z^{(3)}-z^{(1)} & z^{(4)}-z^{(1)}
\end{array}\right|
$$

The averaging surface area $\langle S\rangle$ and volumes $\langle V\rangle$ are obtained from 5000 samples of the tetrahedrons which are initially equilateral with side length $\eta$.

The temporal evolution of the averaging surface area and volume obtained from DNS and LES are shown in Figures 12 and 13 respectively. In DNS, both of these two quantities exhibit little change for $t<3 \tau_{\eta}$ and a rapid monotonic growth for $t>3 \tau_{\eta}$. The result for $t<3 \tau_{\eta}$ can be well predicted by LES but, for $t>3 \tau_{\eta}$, the result from LES grows more slowly than that from DNS. The observation can be understood by investigating the properties of velocity fields.

At the initial stage, the velocity fields are incompressible and thus local velocity fields in both DNS and LES preserve the surface area and volume of any tetrahedrons [9]. As time increases, the tetrahedrons are also advected by the large-scale velocity field. The velocity fluctuations in LES are less than that in DNS. Therefore, both area and volume in LES increase less rapidly than those in DNS, leading to an underprediction of LES on multiparticle dispersion. 


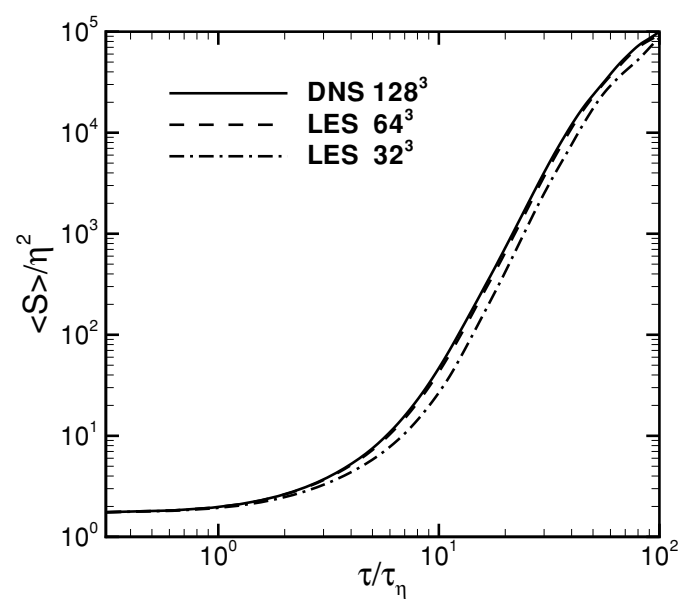

Figure 12. Means of surface areas of tetrahedrons from DNS and LES.

The shape of a tetrahedron can be characterized by the renormalized volume ratio $\lambda_{V}$ and surface area ratio $\lambda_{S}$, defined by

$$
\lambda_{V}=V / S^{3 / 2}, \lambda_{S}=S / C^{2}
$$

where $C$ denotes the perimeter. Since the tetrahedron of a given surface area with maximum volume and the tetrahedron of a given perimeter with maximum surface area are both regular, the regular tetrahedron has the largest ratio and a slender one has a smaller value. Two extreme cases are $\lambda_{V}=0$ corresponding to four particles being coplanar and $\lambda_{S}=0$ to four particles being collinear. Figure 14 plots the temporal evolution of the averaging renormalized volume ratio $\left\langle\Lambda_{V}\right\rangle$ and surface area ratio $\left\langle\lambda_{S}\right\rangle$ of the tetrahedrons from initially regular ones of edge size $\eta$.

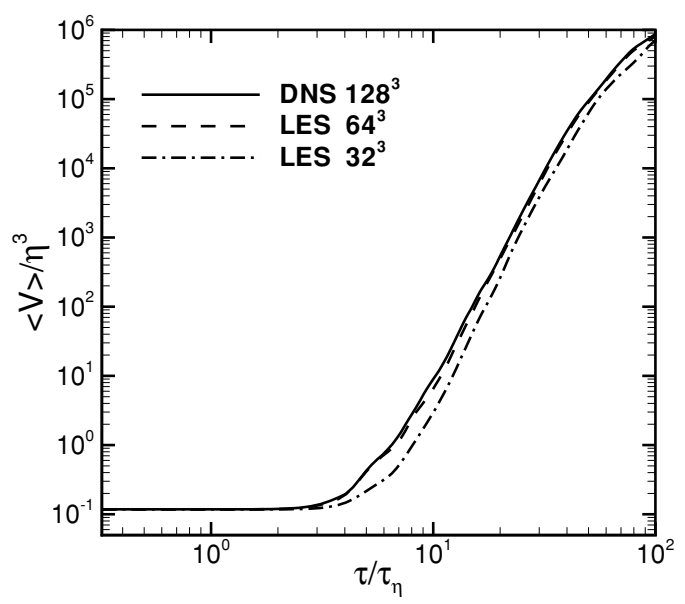

Figure 13. Means of volumes of tetrahedrons from DNS and LES. 


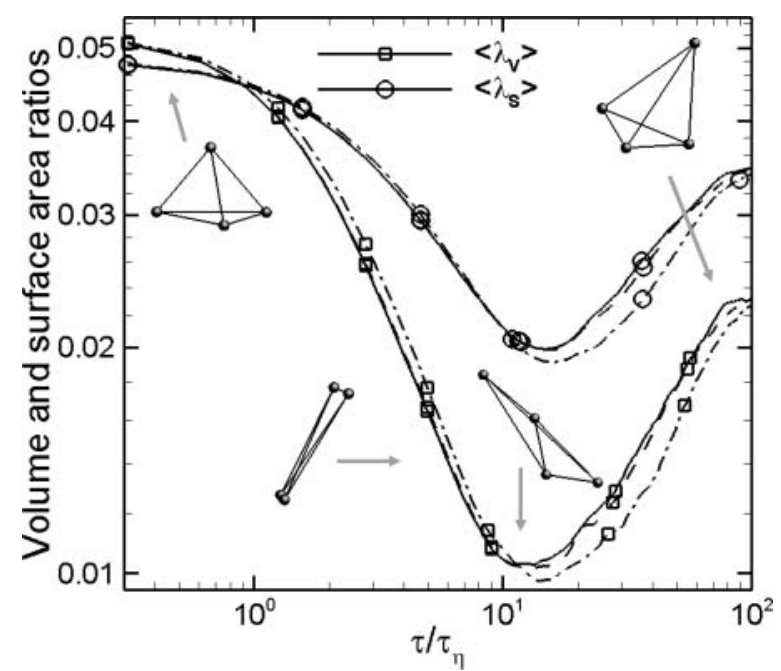

Figure 14. Temporal evolution of averaging renormalized volume and surface area ratios from DNS and LES: solid lines DNS $128^{3}$, dashed lines LES $64^{3}$ and dash-dotted lines LES $32^{3}$. The typical shapes corresponding to the ratios are also indicated along with curves.

At a very short time, the ratios exhibit little changes and the results from DNS and LES are in good agreement with each other. This is because the velocity fields in both DNS and LES can be well approximated locally by the linear maps which preserve both volume and area, as indicated in Figures 12 and 13. At medium time, the ratios drop down to their minimum values due to turbulent stretching. The ratios from LES decrease more slowly than those from DNS, since the velocity field from LES stretches the tetrahedron less intensively. At larger times, the ratios from LES increase more slowly than those from DNS. Here, in comparison with DNS, the distorted tetrahedron in LES is more likely to have at least one edge of length compared with subgrid scales, which is consistent with the observation in Figure 10(b). The particle pair linked by the edge is less dispersed by LES so that the tetrahedron is less diffusive in LES than in DNS. Therefore, the shape change of a tetrahedron in LES is always slower than in DNS. Finally, the curves obtained from DNS and LES appear to converge because the lengths of all edges of a tetrahedron are comparable with the Eulerian integral scale. Therefore, the shape of a tetrahedron is mainly governed by the large-scale motions of turbulent flows.

Another indicator for shape changes was introduced by Pumir et al. [9] and later investigated by Biferale et al. [33]. The indicator is defined as the moment of the intermediate eigenvalue of the inertia matrix $\mathbf{I}=\boldsymbol{\rho} \boldsymbol{\rho}^{T}$, where

$$
\begin{gathered}
\boldsymbol{\rho}=\left(\begin{array}{ccc}
\rho_{x}^{(1)} & \rho_{x}^{(2)} & \rho_{x}^{(3)} \\
\rho_{y}^{(1)} & \rho_{y}^{(2)} & \rho_{y}^{(3)} \\
\rho_{z}^{(1)} & \rho_{z}^{(2)} & \rho_{z}^{(3)}
\end{array}\right), \\
\boldsymbol{\rho}^{(i)}=\left(\rho_{x}^{(i)}, \rho_{y}^{(i)}, \rho_{z}^{(i)}\right), \quad i=1,2,3,
\end{gathered}
$$




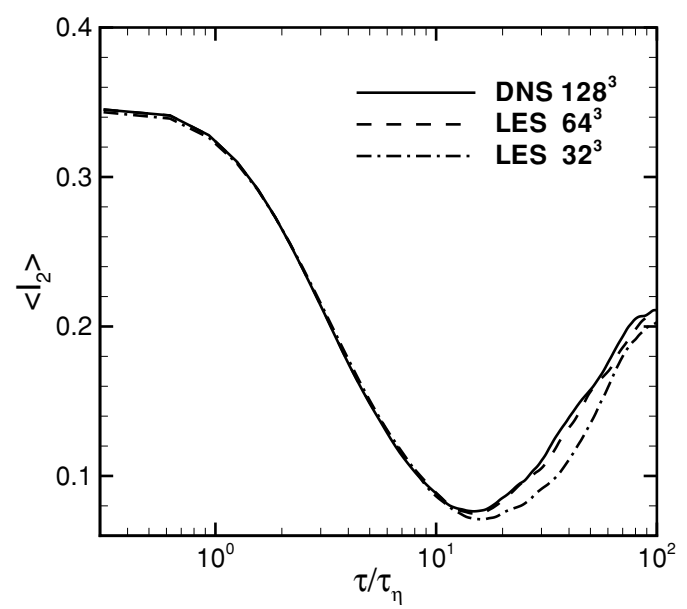

Figure 15. Temporal evolution of the ratio $\left\langle I_{2}\right\rangle$ from DNS and LES.

and

$$
\begin{aligned}
& \rho^{(1)}=\left(\mathbf{x}^{(2)}-\mathbf{x}^{(1)}\right) / \sqrt{2}, \\
& \rho^{(2)}=\left(2 \mathbf{x}^{(3)}-\mathbf{x}^{(1)}-\mathbf{x}^{(2)}\right) / \sqrt{6}, \\
& \rho^{(3)}=\left(3 \mathbf{x}^{(4)}-\mathbf{x}^{(1)}-\mathbf{x}^{(2)}-\mathbf{x}^{(3)}\right) / \sqrt{12} .
\end{aligned}
$$

We denote the three eigenvalues of the inertia matrix $\mathbf{I}$ as $g_{1}, g_{2}$ and $g_{3}$ with $g_{1} \geq g_{2} \geq g_{3}$, and the ratio $I_{i}=g_{i} / G^{2}$ where $G=\sqrt{\operatorname{tr}(\mathbf{I})}$ is the characteristic size of a tetrahedron. It is shown that $I_{1}=I_{2}=I_{3}=\frac{1}{3}$ corresponds to a regular tetrahedron, $I_{3}=0$ corresponds to a four-particle coplanar, and $I_{2}=I_{3}=0$ corresponds to a four-particle collinear. Figure 15 shows the temporal evolution of the ratio $\left\langle I_{2}\right\rangle$ obtained from DNS and LES: the quantity drops initially and then grows up. In comparison with DNS, the result from LES is a little larger for the decreasing $\left\langle I_{2}\right\rangle$ and smaller for the increasing $\left\langle I_{2}\right\rangle$. The observations are in agreement with those from the renormalized volume and surface area ratios in Figure 14.

\section{Prediction of Taylor time micro-scales in DNS and LES}

In this section, we will develop a prediction of the Lagrangian Taylor time micro-scales in DNS and LES using a turbulence closure theory, in order to better understand the observations made in the previous sections. The Taylor micro-scales are calculated from the governing equations of the DNS and LES.

To facilitate the analysis, we assume that the velocity fields in the DNS and LES are identical at an initial time or at the present stage. The Taylor expansions in powers of time $\tau$ of the Eulerian 
and Lagrangian velocity field are written as

$$
\begin{aligned}
\mathbf{u}(\mathbf{x}, t+\tau) & =\sum_{n} \frac{\tau^{n}}{n !} \mathbf{u}^{(n)}(\mathbf{x}), \\
\mathbf{V}(\mathbf{x}, t \mid t+\tau) & =\sum_{n} \frac{\tau^{n}}{n !} \mathbf{V}^{(n)}(\mathbf{x}, t \mid t),
\end{aligned}
$$

where $\mathbf{u}(\mathbf{x}, t)$ is the Eulerian velocity at position $\mathbf{x}$ and time $t, \mathbf{V}(\mathbf{x}, t \mid t+\tau)$ is the Lagrangian velocity at time $t+\tau$ of the fluid particle that was at position $\mathbf{x}$ at time $t$, and the superscript (n) denotes $n$ th-order differentiation with respect to time $\tau$ at fixed $t$. The Taylor time micro-scale of the single-particle Lagrangian velocity correlation is defined as

$$
\tau_{\lambda}^{2}=-\langle\mathbf{V}(\mathbf{x}, t \mid t) \mathbf{V}(\mathbf{x}, t \mid t)\rangle / \frac{\partial^{2}}{\partial \tau^{2}}\langle\mathbf{V}(\mathbf{x}, t \mid t+\tau) \mathbf{V}(\mathbf{x}, t \mid t)\rangle_{\tau=0}
$$

Here, in terms of Kraichnan's generalization [34], the Lagrangian velocity $\mathbf{V}(\mathbf{x}, t \mid t+\tau)$ satisfies

$$
\left(\frac{\partial}{\partial t}+\mathbf{u}(\mathbf{x}, t) \nabla_{\mathbf{x}}\right) \mathbf{V}(\mathbf{x}, t \mid t+\tau)=0
$$

and the Eulerian velocity $\mathbf{u}(\mathbf{x}, t)$ satisfies the Navier-Stokes equation [23]

$$
\begin{aligned}
\frac{\partial \mathbf{u}}{\partial t} & =\mathbf{M u u}+v \Delta \mathbf{u}+\mathbf{s}, \\
2 \mathbf{M a b} & =-(\mathbf{a} \cdot \nabla) \mathbf{b}-(\mathbf{b} \cdot \nabla) \mathbf{a}+2 \mathbf{N a b}, \\
\mathbf{N a b} & =\nabla \nabla^{-2} \frac{\partial a_{j}}{\partial x_{k}} \frac{\partial b_{k}}{\partial x_{j}},
\end{aligned}
$$

where $\mathbf{s}=0$ in DNS and $\mathbf{s}$ is the inverse Fourier transformation of the term corresponding to the spectral eddy-viscosity SGS model in LES. In Equation (24), we have already used the continuity equation to eliminate the pressure term. The forcing term in Equation (1) can be ignored without changing our results [5].

The first two non-vanishing coefficients in the Taylor expansion can be calculated by following the method described in [5] and [23]

$$
\begin{aligned}
C_{0} & \equiv\langle\mathbf{V}(\mathbf{x}, t \mid t) \mathbf{V}(\mathbf{x}, t \mid t)\rangle=\langle\mathbf{u}(\mathbf{x}, t) \mathbf{u}(\mathbf{x}, t)\rangle \\
C_{2} & \equiv \frac{\partial^{2}}{\partial \tau^{2}}\langle\mathbf{V}(\mathbf{x}, t \mid t+\tau) \mathbf{V}(\mathbf{x}, t \mid t)\rangle_{\tau=0}, \\
& =\langle[2 \mathbf{N u}(\mathbf{M u u})+(\mathbf{u} \cdot \nabla)(\mathbf{N u u})] \cdot \mathbf{u}\rangle+v^{2}\left\langle\Delta^{2} \mathbf{u} \cdot \mathbf{u}\right\rangle+\langle\mathbf{s} \cdot \mathbf{u}\rangle .
\end{aligned}
$$


Using EDQNM theory in Fourier space, we can calculate the coefficients in DNS

$$
\begin{aligned}
& C_{0}^{\mathrm{DNS}}=2 \int_{0}^{\infty} E(k) \mathrm{d} k, \\
& C_{2}^{\mathrm{DNS}}=-2 \int_{0}^{\infty} k E(k) \mathrm{d} k \int_{0}^{k} q E(q) j(q / k) \mathrm{d} q+v^{2} \int_{0}^{\infty} k^{4} E(k) \mathrm{d} k .
\end{aligned}
$$

where the geometric factor $j(q / k)$ is always positive [23]. Similarly, we can obtain the coefficients in LES

$$
\begin{aligned}
C_{0}^{\mathrm{LES}} & =2 \int_{0}^{k_{c}} E(k) \mathrm{d} k, \\
C_{2}^{\mathrm{LES}} & =-2 \int_{0}^{k_{c}} k E(k) \mathrm{d} k \int_{0}^{k} q E(q) j(q / k) \mathrm{d} q+\int_{0}^{k_{c}}\left[v^{2}+v_{t}^{2}\left(k \mid k_{c}\right)\right] k^{4} E(k) \mathrm{d} k .
\end{aligned}
$$

If the Reynolds number is very high, the molecular viscous terms can be negligible. Comparison of Equation (30) with Equation (32) yields

$$
C_{2}^{\mathrm{LES}}=C_{2}^{\mathrm{DNS}}+2 \int_{k_{c}}^{\infty} k E(k) \mathrm{d} k \int_{0}^{k} q E(q) j(q / k) \mathrm{d} q+\int_{0}^{k_{c}} v_{t}^{2}\left(k \mid k_{c}\right) k^{4} E(k) \mathrm{d} k .
$$

Then $C_{2}^{\mathrm{DNS}}<C_{2}^{\mathrm{LES}}$ or $-C_{2}^{\mathrm{DNS}}>-C_{2}^{\mathrm{LES}}$, since the last two terms in Equation (33) are positive. In an ideal LES, we have $C_{0}^{\mathrm{DNS}}=C_{0}^{\mathrm{LES}}$. Then,

$$
\tau_{\lambda}^{\mathrm{LES}}>\tau_{\lambda}^{\mathrm{DNS}}
$$

This result is consistent with the numerical observation that the Taylor micro-scale in the LES is always larger than that in the DNS.

Equation (33) also implies that $C_{2}^{\mathrm{LES}}-C_{2}^{\mathrm{DNS}}$ is a decreasing function of the cutoff wavenumber $k_{c}$, since the second term on the right-hand side (r.h.s.) of the equation is the decreasing function of $k_{c}$ and the third term changes slightly. As expected, increasing the resolution of LES could improve the predicted Taylor micro-scales. Furthermore, if LES resolves most of energy in DNS, both the second and third terms will be very small. The Taylor micro-scale will be well predicted. In our numerical experiments, the results from $N=64$ are better than those from $N=32$ since the former resolves the energy spectrum more fully than the latter.

Those discrepancies between DNS and LES can be attributed to the absence of small-scale motions and the function of the eddy-viscosity SGS model in the LES, as indicated by the last two terms in Equation (33). The second term on the r.h.s. of Equation (33) represents the effect of small-scale motions, which are unresolved in LES. The small-scale eddies fluctuate intensively and enhance particle dispersion. The third term on the r.h.s. of Equation (33) represents the effects of the SGS model on Lagrangian velocity correlations. The SGS model dissipates energy to balance the energy input. However, it acts as a dissipation source and smoothes down the intermittency, which produces a more correlated velocity field. This analysis can be further applied to identify the contributions of subgrid scale motions to Lagrangian time correlations. Unlike the time correlations of Eulerian velocity modes [5], filtering alone does alter the Lagrangian time correlations, which is in agreement with a priori test made by Fede and Simonin [21]. 


\section{Discussions and conclusions}

A variety of Lagrangian statistics are computed from DNS and LES flow fields for homogeneous isotropic turbulence. The LES makes use of a spectral eddy-viscosity model. For the case of longtime single-particle dispersion, it is shown that, compared to DNS, LES overpredicts the time scale of the Lagrangian velocity correlation but underpredicts the Lagrangian velocity fluctuation. The two effects tend to cancel one another leading to an accurate prediction of the longtime turbulent dispersion coefficient. This is intuitively consistent with the picture that the longtime turbulent dispersion is governed by large-scale motion of the fluid turbulence whose dynamics is adequately represented in LES.

Unlike the single-particle dispersion, the particle pair dispersion at dissipation-range scales is influenced more by small-scale velocity fluctuations. It is shown that LES with a typical eddyviscosity model tends to underestimate the rate of relative dispersion due to the lack of SGS fluctuations. Namely, the pair dispersion at small separation is enhanced by small-scale motions which are not explicitly included in LES. The difference between LES and DNS results on pair dispersion depends on the cutoff scale in LES, and is negligible when the initial separation of the pair is made comparable to the resolved scales in LES.

Similar results are found regarding multiparticle dispersion in which both the size and topological shape of clusters are considered. It is shown that the average surface area and volume of a tetrahedron of particle quadruplet increase more slowly in the LES than those in the DNS. This is due to the lack of effective straining motion in LES at dissipation-range scales, and consequently a longer time is required for multiparticle clusters with initial size in the dissipation range to develop a length scale comparable to inertial-range scales. For the same reason, the topologically shape changes of particle clusters in LES are always slower than those in DNS.

A turbulence closure theory is used to better understand the differences in Lagrangian statistics between LES and DNS. Starting from the governing equations for DNS and LES, we develop a theoretical representation for the Taylor time micro-scale of the Lagrangian velocity correlations. The results from theoretical approximations confirm the numerical observation that the Taylor micro-scale is larger in LES than that in DNS. The theory indicates that the overprediction of the time scale of the Lagrangian velocity correlations in LES results from both the absence of SGS motions and the eddy-viscosity model. Interestingly, we found that the eddy-viscosity SGS model increases the time scale of the Lagrangian velocity correlations. Namely, it makes an additional negative contribution to the accurate prediction of Lagrangian statistics in LES though it plays a positive role in predicting Eulerian spectra. This is also in agreement with our previous observation on Eulerian time correlations [5].

The simulation and theoretical results suggest that the LES with an eddy-viscosity model could significantly overpredict the time scale of the Lagrangian velocity correlations and underpredict turbulent relative dispersion. Such a SGS model makes little or even negative contribution to the LES prediction on Lagrangian velocity correlations, since the contribution of unresolved Lagrangian velocity fields to Lagrangian statistics could not be recovered from resolved Eulerian velocity fields. Moreover, the effects of small-scale motions unsolved in LES on particle dispersion cannot be represented by the eddy-viscosity SGS models, which suppress the particle dispersion. To recover the contribution of the unresolved Lagrangian velocity field, a SGS model for Lagrangian velocity field needs to be developed.

\section{Acknowledgment}

This work was supported by Chinese Academy of Sciences under the innovative project 'Multi-scale modelling and simulation in complex systems' (KJCX-SW-L08) and National Natural Science Foundation 
of China under the project nos. 10325211 and 10628206. LPW acknowledges support from US National

Science Foundation (ATM-0527140) and the National Center for Atmospheric Research (NCAR).

\section{References}

[1] P. Moin, Advances in large-eddy simulation methodology for complex flows, Int. J. Heat. Fluid. Flow. 23 (2002), p. 710.

[2] F. Mashayek and R.V.R. Pandya, Analytical description of particle/droplet-laden turbulent flows, Prog. Energy Combust. Sci. 29 (2003), p. 329.

[3] B. Sawford, Turbulent relative dispersion, Annu. Rev. Fluid Mech. 33 (2001), p. 289.

[4] P.K. Yeung, Lagrangian investigations of turbulence, Annu. Rev. Fluid Mech. 34 (2002), p. 115.

[5] G.-W. He, R. Rubinstein, and L.-P. Wang, Effects of subgrid-scale modeling on time correlations in large eddy simulation, Phys. Fluids 14 (2002), p. 2186.

[6] G.-W. He, M. Wang, and S.K. Lele, On the computation of space-time correlations by large-eddy simulation, Phys. Fluids 16 (2004), p. 3859.

[7] G.I. Taylor, Diffusion by continuous movement, Proc. Lond. Math. Soc. 20 (1921), p. 196.

[8] G.K. Batchelor, Diffusion in a field of homogeneous turbulence. II. The relative motion of particles, Proc. Camb. Phil. Soc. 48 (1952), p. 345.

[9] A. Pumir, B.I. Shraiman, and M. Chertkov. Geometry of Lagrangian dispersion in turbulence, Phys. Rev. Lett. 85 (2000), p. 5324.

[10] Q. Wang, K.D. Squires, and X. Wu, Lagrangian statistics in turbulent channel flow, Atmos. Environ 29 (1995), p. 2417.

[11] Q. Wang and K.D. Squires, Large eddy simulation of particle-laden turbulent channel flow, Phys. Fluids 8 (1996), p. 1207.

[12] V. Armenio, U. Piomelli, and V. Fiorotto, Effect of subgrid scales on particle motion, Phys. Fluids 11 (1999), p. 3030.

[13] J. Zhang, G.-W.He, and L.P. Lu, Subgrid scale contributions to Lagrangian velcoity correlations in isotropic turbulence, International Workshop on Wither Turbulence Prediction and Control, Korea, Mar. 26-29, 2006.

[14] G. Wei et al. Scalar dispersion by a large-eddy simulation and a Lagrangian stochastic subgrid model, Phys. Fluids 18 (2006), 095101.

[15] F. Yeh and U. Lei, On the motion of small particles in a homogeneous isotropic turbulent flow, Phys. Fluids A 3 (1991), p. 2571.

[16] C.Y. Yang and U. Lei The role of the turbulent scales in the settling velocity of heavy particles in homogeneous isotropic turbulence, J. Fluid Mech. 371 (1998), p. 179.

[17] B. Shotorban and F. Mashayek, Modeling subgrid-scale effects on particles by approximate deconvolution, Phys. Fluids 17 (2005), 081701.

[18] J.G.M. Kuerten, Subgrid modeling in particle-laden channel flow, Phys. Fluids 18 (2006), 025108 .

[19] J.G.M. Kuerten and A.W. Verman, Can turbuphoresis be predicted by large-eddy simulation?, Phys. Fluids 17 (2005), 011701.

[20] B. Shotorban and F. Mashayek, A stochastic model for particle motion in large-eddy simulation, J. Turbulence. 7 (2006), 1.

[21] P. Fede and O. Simonin, Numerical study of the subgrid fluid turbul effects on the statistics of heavy colliding particles, Phys. Fluids 18 (2006), 045103.

[22] R.H. Kraichnan, Convergents to turbulent functions, J. Fluid Mech. 41 (1970), p. 189.

[23] Y. Kaneda and T. Gotoh, Lagrangian velocity autocorrelation in isotropic turbulence, Phys. Fluids A 3 (1991), p. 1924.

[24] Y. Kaneda, Lagrangian and Eulerian time correlations in turbulence, Phys. Fluids A 5 (1993), p. 2835.

[25] L.P. Wang and M.R. Maxey, Settling velocity and concentration distribution of heavy particles in homogeneous isotropic turbulence, J. Fluid Mech. 256 (1993), p. 27.

[26] S. Ghosal, An analysis of numerical errors in large-eddy simulations of turbulence, J. Comp. Phys. 125 (1996), p. 187.

[27] J.-P. Chollet and M. Lesieur, Parameterization of small scales of three-dimensional isotropic turbulence utilizing spectral closure, J. Atmos. Sci. 38 (1981), p. 2747. 
[28] S. Balachandar and M.R. Maxey, Methods for evaluation fluid velocities in spectral simulations of turbulence, J. Comp. Phys. 83 (1989), p. 96.

[29] P.K. Yeung and S.B. Pope, An algorithm for tracking fluid particles in numerical simulations of homogeneous turbulence, J. Comp. Phys. 79 (1988), p. 373.

[30] S. Corrsin, Mechanique de la Turbulence, Colloques internationaux du Centre National de la Recherche Scientifique, No. 108 C. N. R. S., Paris, 1961, pp. 27-52.

[31] T. Ishihara and Y. Kaneda, Relative diffusion of a pair of fluid particles in the inertial subrange of turbulence, Phys. Fluids 14 (2002), L69.

[32] P.K. Yeung, Direct numerical simulation of two-particle relative diffusion in isotropic turbulence, Phys. Fluids 6 (1994), p. 3416.

[33] L. Biferale et al., Multiparticle dispersion in fully developed turbulence, Phys. Fluids 17 (2005), 111701.

[34] R.H. Kraichnan, Lagrangian-history closure approximation for turbulence, Phys. Fluids 8 (1965), p. 575 .

\section{Appendix 1. Particle tracking algorithm}

A fluid particle is governed by Equation (5). Usually, the locations of fluid particles are not coincident with the grid nodes in physical space. Therefore, an interpolation algorithm is needed to calculate the Lagrangian velocity $\mathbf{V}\left(\mathbf{x}_{0}, t_{0} \mid t\right)$ at location $\mathbf{X}\left(\mathbf{x}_{0}, t_{0} \mid t\right)$ from the Eulerian velocity field $\mathbf{u}(\mathbf{x}, t)$. The interpolation algorithm used in the present study is a three-dimensional sixth-order Lagrangian interpolation scheme [28]. In the scheme, the Lagrangian velocities are calculated from

$$
\mathbf{V}\left(\mathbf{x}_{0}, t_{0} \mid t\right)=\sum_{i=1}^{N} \sum_{j=1}^{N} \sum_{k=1}^{N} \mathbf{u}\left(x_{i}, y_{j}, z_{k}, t\right) L_{i}(X) L_{j}(Y) L_{k}(Z)
$$

where $\left(x_{i}, y_{j}, z_{k}\right)$ denotes the location of grids node in the Eulerian coordinate system and $(X, Y, Z)$ the coordinate of one fluid particle in the same coordinate system. The basis functions $L_{i}, L_{j}$ and $L_{k}$ are given as the sixth-order Lagrangian polynomials

$$
\begin{aligned}
& L_{i}(x)=0 \text {; } \\
& =\frac{1}{120}\left(4 \xi-5 \xi^{3}+\xi^{5}\right), \\
& \xi=\frac{\left(x-x_{i-3}\right)}{d x} ; \quad x_{i-3} \leq x \leq x_{i-2} \\
& =\frac{1}{24}\left(-6 \xi-\xi^{2}+7 \xi^{3}+\xi^{4}-\xi^{5}\right), \\
& \xi=\frac{\left(x-x_{i-2}\right)}{d x} ; \quad x_{i-2} \leq x \leq x_{i-1} \\
& =\frac{1}{12}\left(12 \xi+8 \xi^{2}-7 \xi^{3}-2 \xi^{4}+\xi^{5}\right), \\
& \xi=\frac{\left(x-x_{i-1}\right)}{d x} ; \quad x_{i-1} \leq x \leq x_{i} \\
& =\frac{1}{12}\left(12-4 \xi-15 \xi^{2}+5 \xi^{3}+3 \xi^{4}-\xi^{5}\right), \\
& \xi=\frac{\left(x-x_{i}\right)}{d x} ; \\
& x_{i} \leq x \leq x_{i+1} \\
& =\frac{1}{24}\left(-12 \xi+16 \xi^{2}-\xi^{3}-4 \xi^{4}+\xi^{5}\right), \\
& \xi=\frac{\left(x-x_{i+1}\right)}{d x} ; \\
& x_{i+1} \leq x \leq x_{i+2} \\
& =\frac{1}{120}\left(6 \xi-5 \xi^{2}-5 \xi^{3}+5 \xi^{4}-\xi^{5}\right), \\
& \xi=\frac{\left(x-x_{i+2}\right)}{d x} ; \quad x_{i+2} \leq x \leq x_{i+3} \\
& =0 \text {; } \\
& x_{i+3} \leq x .
\end{aligned}
$$


Each sixth-order Lagrangian polynomial requires the Eulerian velocities at six grid nodes. Consequently, the evaluation of Lagrangian velocities of $N_{p}$ fluid particles requires the operation of the order $3 \times 6^{3} \times N_{p}$.

The trajectory of an individual fluid particle can be obtained by numerical integration of Equation (5) using the explicit fourth-order Adams-Bashforth method. The time step used here is the same as that used for the Navier-Stokes equations. The calculation requires the storage of the variables at the previous three steps. At the initial stage, explicit Euler, second-order and third-order Adams-Bashforth methods are used for the first three steps, respectively. 